\title{
Spatial Control of Reflexes, Posture and Movement in Normal Conditions and after Neurological Lesions
}

\author{
by \\ Anatol G. Feldman ${ }^{1,3}$, Mindy F. Levin 2,3
}

\begin{abstract}
Control of reflexes is usually associated with central modulation of their sensitivity (gain) or phase-dependent inhibition and facilitation of their influences on motoneurons (reflex gating). Accumulated empirical findings show that the gain modulation and reflex gating are secondary, emergent properties of central control of spatial thresholds at which reflexes become functional. In this way, the system pre-determines, in a feedforward and task-specific way, where, in a spatial domain or a frame of reference, muscles are allowed to work without directly prescribing EMG activity and forces. This control strategy is illustrated by considering reflex adaptation to repeated muscle stretches in healthy subjects, a process associated with implicit learning and generalization. It has also been shown that spasticity, rigidity, weakness and other neurological motor deficits may have a common source - limitations in the range of spatial threshold control elicited by neural lesions.
\end{abstract}

Key words: motor control, rehabilitation, stretch reflex, spasticity, learning.

\section{Introduction}

Central control of proprioceptive reflexes such as the stretch reflex and intermuscular reflexes is usually associated with modulation of their sensitivity (gain) to changes in the position and velocity of body segments or as reflex gating - phase-dependent inhibition and facilitation of reflex pathways to $\alpha$-MNs, as is often observed during locomotion (Evarts and Tanji, 1974; Sillar, 1991; Wei et al., 2014). Reflex position- and velocity-dependent reactions contribute to stability of posture and together with intrinsic muscle properties comprise posture-stabilizing mechanisms. The concept of reflex gating implies that proprioceptive and cutaneous afferent feedback to motoneurons (MNs) may or may not be suppressed depending on the phase of motion. Gain modulation and reflex gating are mediated by spinal and descending systems via pre-, post-, mono- and poly-synaptic influences on $\alpha$-MNs or indirectly, via $\gamma$-MNs (Hultborn, 2006; Stein and
Capaday, 1988; for recent review see Feldman, 2016).

Experimental data starting from those obtained by Matthews (1959) and Asatryan and Feldman (1965) suggest that gain modulation and reflex gating might be an emergent property of a primary central process called the spatial control of muscle activation (Turpin et al., 2016). The general idea is that, in the intact system, central structures specify where, in a spatial domain or a frame of reference (FR), MNs can be activated without specific instructions of how they should be activated. MN activation emerges following the interaction of the homonymous muscle with external forces if afferent feedback indicates that the muscle works within the spatial FR predetermined by central inputs. Indeed, this notion conflicts with the conventional view that muscle activation is basically pre-determined by central pattern generators (CPGs). The notion of spatial

1 - Department of Neuroscience, University of Montreal, Montreal, Quebec, Canada.

2 - School of Physical and Occupational Therapy, McGill University, Montreal, Quebec, Canada.

3 - Centre for Interdisciplinary Research in Rehabilitation (CRIR), Montreal, Quebec, Canada. 
control is well justified empirically and helps solve several problems in action and perception that remain unexplained in the conventional framework. In particular, the classical posturemovement problem of how intentional movement can be produced without evoking resistance of posture-stabilizing mechanisms (von Holst, 1954; Stuart, 2005) is solved in the framework of spatial control (Feldman, 2016).

Spatial control of $\alpha$-MNs has been described in several publications (e.g. Feldman, 1986, 2016; Turpin et al., 2016). In brief, taken together, anatomical and neurophysiological data justify the following condition of muscle activation (Figure 1): if $x$ is the current muscle length, then the muscle is active if

$$
x-\lambda^{*}>0
$$

where $\lambda^{*}$ is the dynamic threshold muscle length:

$$
\lambda^{*}=\lambda-\mu v+Q
$$

which means that $\lambda^{*}$ decreases with stretch velocity ( $\mathrm{v}>0$ for muscle stretching and $\mathrm{v}<0$ for shortening); $\mu$ is the sensitivity of the threshold to velocity that possibly can be controlled by dynamic $\gamma$-MNs. Term $\mathrm{Q}$ is a combined measure of the influence of intermuscular interactions and the history-dependent intrinsic state of MNs (e.g., associated with plateau potentials). In the suprathreshold range, recruitment of motor units and active muscle force increase with the difference between the actual and threshold muscle lengths. Threshold $\lambda^{*}$ defines the range of muscle lengths in which active muscle force can be generated.

These formulas account for different ways by which muscles can be activated or deactivated. In particular, the threshold length $\left(\lambda^{*}\right)$ can be considered as the threshold of the stretch reflex. This does not mean that this reflex is the only way of activating a muscle: the initially silent muscle can be activated centrally, by decreasing the activation threshold. The initially silent muscle can also be activated transiently, by brief rapid stretching, as occurs during the tendon jerk reflex. On the other hand, rapid transient shortening of an initially active muscle can elicit a silent period in its EMG activity. This phasic reaction, as well as the tendon jerk reflex, indicates that the activation threshold is velocitydependent (equation 2). Responses to muscle stretches can be prevented by increasing the spatial threshold (Turpin et al., 2016). By increasing the threshold, the stretch reflex is not suppressed as such - it remains fully functional, but at longer muscle lengths. Therefore, gating of reflexes should be considered as an emergent property of the motor control system caused by shifts in spatial thresholds, rather than by direct timing of reflexes by CPGs. Only when the threshold is shifted beyond the upper biomechanical limit of the range of muscle lengths will the muscle be relaxed within the entire biomechanical range. When the threshold lies beyond this upper limit, the muscle can only be activated by stretching at a high speed, as occurs during the tendon jerk reflex. The muscle can be activated at the biomechanically shortest length by setting the threshold below that length. Taken together, these properties imply that the normal range of the central regulation of the threshold length $\left(\lambda_{-} \lambda_{+}\right)$exceeds the biome-chanical range of the actual muscle length $\left(x_{-}, x_{+}\right)$(Figure 1). This principle may be violated after damage to the central nervous system, resulting in spasticity, weakness and movement incoordination (see below, Levin et al., 2000; Mullick et al., 2013).

Some descending systems can shift the electrical threshold, $\mathrm{V}_{+}$, of $\alpha$-MNs (Fedirchuk and Dai, 2004; Heckman et al., 2008; Krawitz et al., 2001). A testable prediction is that this might be an additional mechanism of central changes in the spatial threshold, $\lambda$. It is also possible that, compared to low-fatigable motor units, fastfatigable motor units have greater dynamic sensitivity $(\mu)$. As a consequence, with increasing speed of a stretch of a muscle, these motor units can be recruited before low-fatigable motor units (recruitment reversal), a prediction that can also be tested.

Threshold position control is important for transferring balance and stability from one body posture to another, an integral part of practically all intentional (self-initiated) movement and isometric torque production. For example, locomotion can be defined as resulting from translation of body balance and stability in the environment. Reach-to-grasp, sit-to-stand movements, jumping and pushing the hand against a wall also require shifting body balance and stability from an initial to a final body posture (Feldman, 2015). 

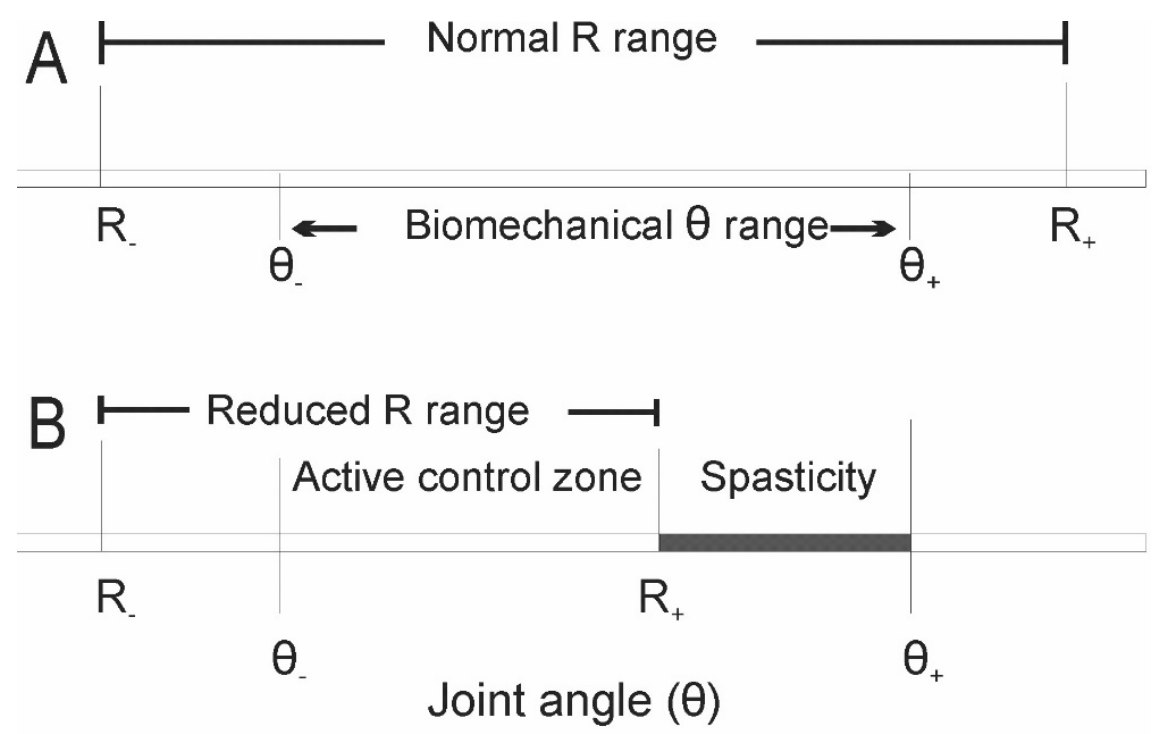

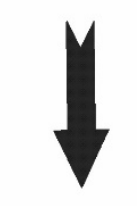

Normal
motor
control
Spasticity

Weakness

Disordered

mtor control

\section{Figure 1}

Central control of spatial thresholds of proprioceptive reflexes and motor deficits resulting from limitations in the range of threshold control. The threshold muscle length $(\lambda)$ corresponds to the joint angle $(R)$ at which motoneurons begin to be recruited. Conditionally, flexor muscles lengthen and extensor muscles shorten with an increasing joint angle. A: Normally, the range $\left(R_{-}, R_{+}\right)$of threshold regulation exceeds the biomechanical range of the joint $\left(\theta_{-}, \theta_{+}\right)$, thus enabling the system to relax or activate muscles at any angle within the biomechanical range. B: Following a brain lesion, the upper limit $\left(R_{+}\right)$of threshold regulation (called Tonic Stretch Reflex Threshold or TSRT, see Figure 5) can fall within the biomechanical range, resulting in an inability to relax flexor muscles at joint angles exceeding $R_{+}$. Clinically, this deficit in post-stroke subjects is identified as spasticity (spasticity zone). In the area below the upper limit (active control zone), the ability to change the muscle force and position via threshold regulation is preserved. Thus, impairment after a brain lesion (spasticity, weakness, disordered motor control) results from a deficit in the specification and regulation of TSRTs. This is a conceptual departure from consideration of each symptom as an individual impairment, unrelated to the others. In the zone between $\theta$. and $R_{+}=T S R T_{+}$, normal patterns of muscle activation are possible. Movements attempted in the zone between TSRT+ and $\theta_{+}$are accompanied by spasticity and weakness as well as disordered muscle activation patterns. 
A physical principle formulated in the dynamic systems theory, as well as in thermodynamic theory of stability (Andronov and Chaikin, 1949; Glansdorf and Prigogine, 1971), stipulates that the specific choice of body posture at which balance and stability can be achieved is not defined by forces, torques or other variables that describe the motor outcome, including EMG patterns related to muscle forces. The choice is defined by parameters that can be changed independently of those variables. The threshold position $(\lambda)$ is an experimentally identified parameter underlying shifts in the equilibrium position of body segments, a justification of the equilibrium-point hypothesis (Asatryan and Feldman, 1965; Feldman, 1986) extended to the theory of referent (threshold) control of action and perception (Feldman, 2015). It follows from this framework that, since motor commands (EMG patterns) are not responsible for shifts in the equilibrium position, their direct specification would be met with resistance of posturestabilizing mechanisms. In other words, the notion of direct programming of motor commands is associated with the classical posture-movement problem (von Holst, 1954; Stuart, 2005). In contrast, by shifting spatial muscle activation thresholds, the system transfers balance and stability to a new equilibrium point and motor output, including motor commands, emerges without any pre-programming following the natural tendency of the system to reach the new equilibrium, attractor point. Parametric control thus allows the nervous system to convert posture-stabilizing to movement producing mechanisms without any posture-movement problem.

Threshold control may underlie movement production, learning and generalization in healthy subjects. In addition, we show that deficits in threshold control account for problems in the control of posture and movement in patients with lesions in the central nervous system.

\section{Normal threshold control of reflexes}

Consider several comparatively simple examples of threshold control in healthy subjects. One of them is the attenuation of reflex responses to repeated stretching of pre-activated muscles (Horak et al., 1989; Nashner, 1976; Turpin et al.,
2016; Rothwell et al., 1986). In a recent study (Turpin et al., 2016), the forearm of healthy subjects was placed on a horizontal manipulandum. Elbow flexors or extensors compensated an external load and were stretched by $20^{\circ}$ or $70^{\circ}$ rotations. Participants were instructed not to intervene by intentionally modifying the muscle resistance elicited by stretching. In addition to phasic stretch reflexes (SRs), muscle stretching was associated with inhibitory periods (IPs) in the ongoing muscle activity starting at a minimal latency of $35 \mathrm{~ms}$ (Figure 2). The SR amplitude decreased dramatically across 5-12 trials and was not restored after a resting period of 3-5 min, despite the increase in stretch amplitude from 20 to $70^{\circ}$. Despite the attenuation of SR responses, inhibitory periods remained present. When SRs were suppressed, stretching of originally nonstretched, antagonist muscles initiated after the rest period showed immediate SR suppression while IPs remained present in the first and subsequent trials (Figure 3). These results suggest that adaptation to muscle stretching includes features characteristic of implicit learning such as memory consolidation and generalization.

Based on the notion of SR threshold control, these results can be explained in the following way. In the study by Turpin et al. (2016), muscles to be stretched were pre-activated to counteract the initial load. This can be achieved by setting $\lambda$ below the muscle length at the initial elbow position $(x-\lambda>0$; Figure 4$)$. Anticipating muscle stretching, the system could prepare to increase and trigger these threshold shifts as soon as stretching started. Prepared at the level of spinal interneurons responsible, in particular, for pre-synaptic inhibition of MNs, the threshold shifts could be produced sufficiently early to attenuate phasic SR responses to muscle stretching. Produced rapidly, shifts in $\lambda$ could overrun the ongoing muscle stretching, suppressing not only SR responses, but the ongoing EMG activity and thus eliciting an IP.

Figure 4 shows three patterns of threshold shift timing. In pattern a, descending systems maintain the same value of $\lambda$ and let the peripheral mechanisms generate phasic and tonic SR responses (Figure 4B). The latter as well as muscle resistance will monotonically increase until muscle stretching is ceased. In pattern $\mathbf{b}$, 
descending systems accomplish a rapid and large monotonic increase in $\lambda$ as soon as stretching starts, attenuating SR responses and suppressing the ongoing muscle activity during the entire stretch, thus producing an IP. Pattern c consists of several ramp-and-hold-like increases in $\lambda$, resulting in attenuation of phasic SR responses and several IPs. During each holding phase of $\lambda$, the stretched muscle reaches and exceeds the activation threshold and the muscle is reactivated. Multiple IPs, thus allow the system gradually, in several steps, to yield to the muscle stretching while minimizing muscle active resistance during and at the end of stretching. This qualitatively explains the occurrence of a single prolonged IP and multiple IPs with muscle activation between them in the study by Turpin et al. (2016). The multi-step pattern of shifts in the activation thresholds could be applied simultaneously to MNs of both stretched and shortened muscles. This common pattern could be used to produce adaptive responses to the first and subsequent stretches of previously non-stretched muscles (generalization of learning).

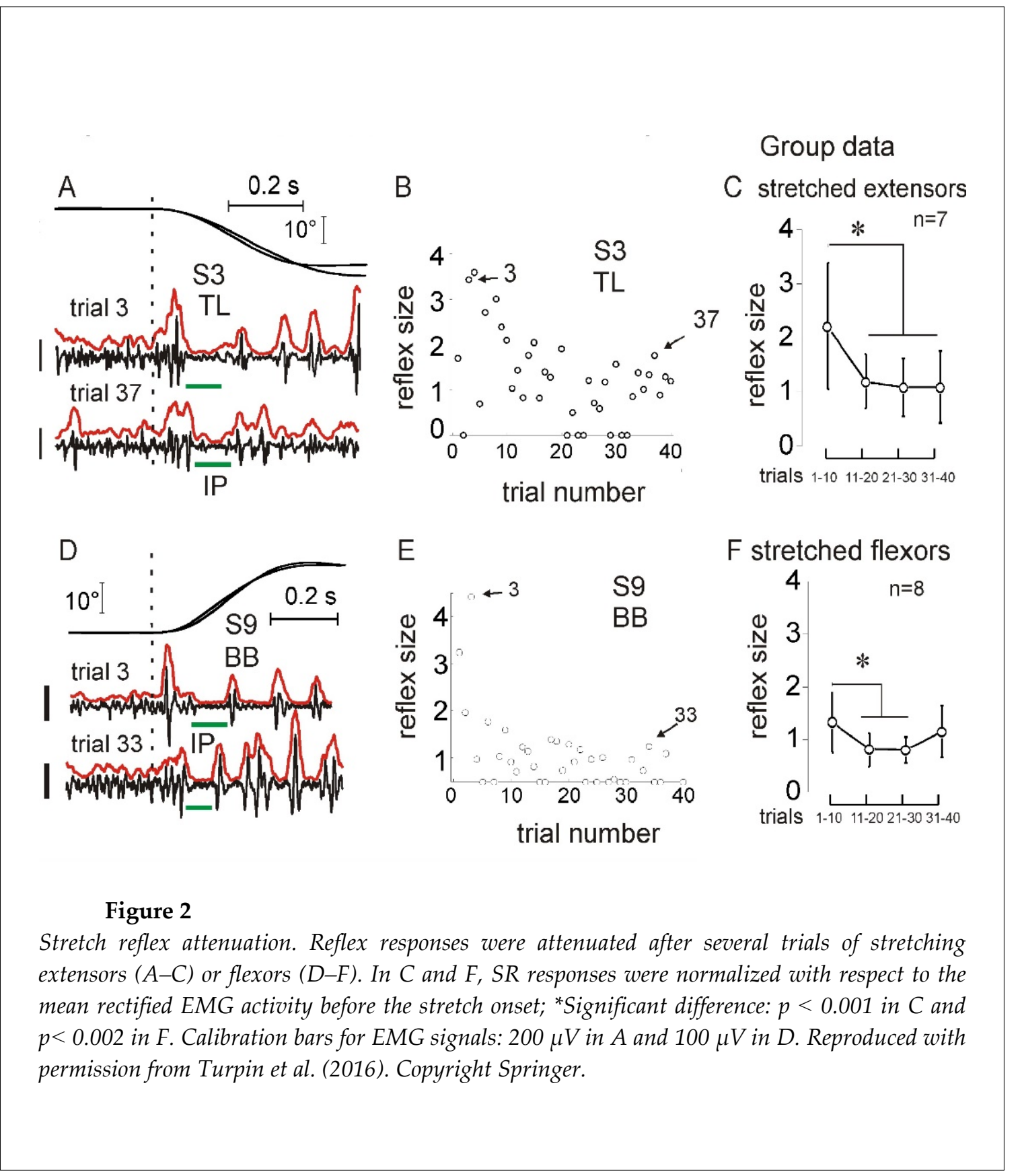

(C) Editorial Committee of Journal of Human Kinetics 


\section{Generalization of learning}

Block 2
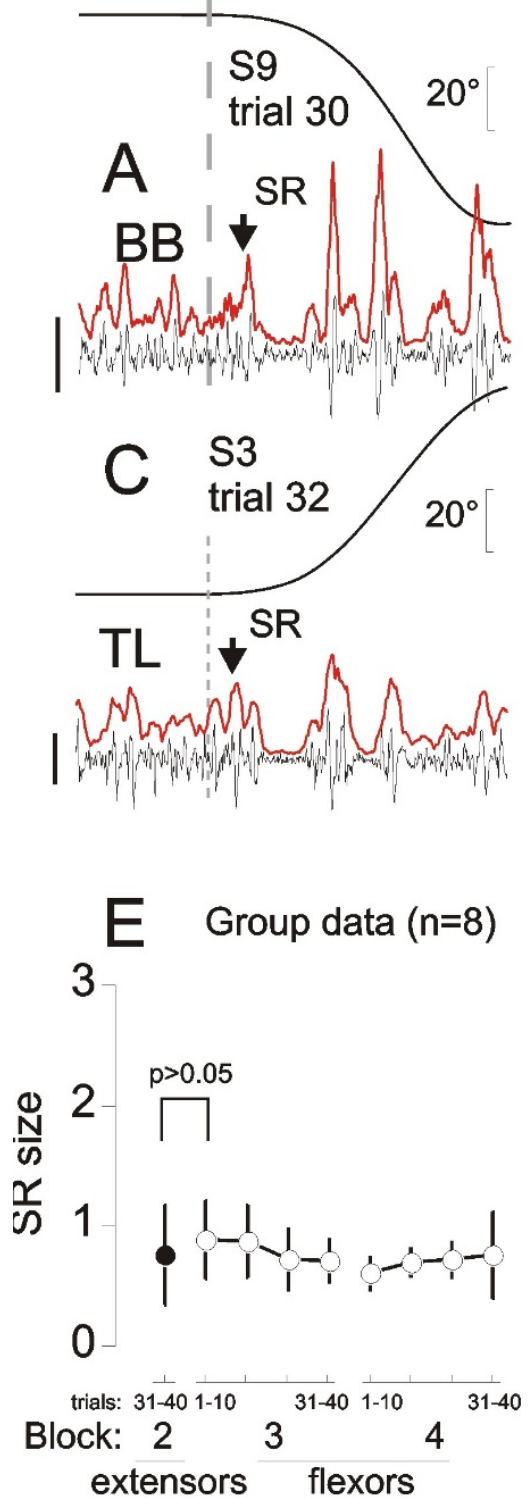

Block 3
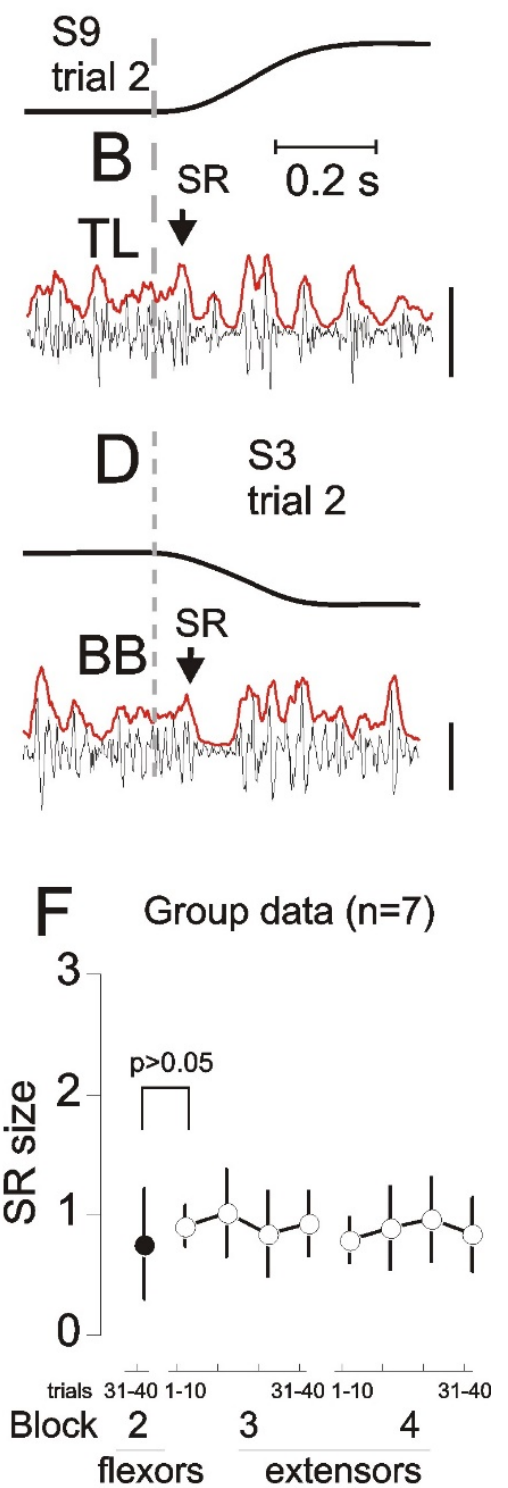

Figure 3

Generalization of SR attenuation. A: example of flexor stretch reflex (SR) response (arrow) and the inhibitory period (IP) from one of the trials in which SRs were attenuated. B: after a 3 to $5 \mathrm{~min}$ rest period, originally non-stretched extensors began to be stretched. Extensor SR was immediately suppressed (arrow) without preliminary trials of stretching. $C$ and $D$ : a similar effect of flexor SR suppression was observed after previous suppression of extensor SRs. E and F: group dataantagonist SRs were suppressed without preliminary attenuation if SRs of agonist muscles were suppressed during repeated stretches before a 3 to 5 min rest period. Calibration bars for EMG signals: $100 \mu \mathrm{V}$ ( $A$ and $B$ ) and $200 \mu V$ (C and D). Reproduced with permission from Turpin et al. (2016). Copyright Springer. 


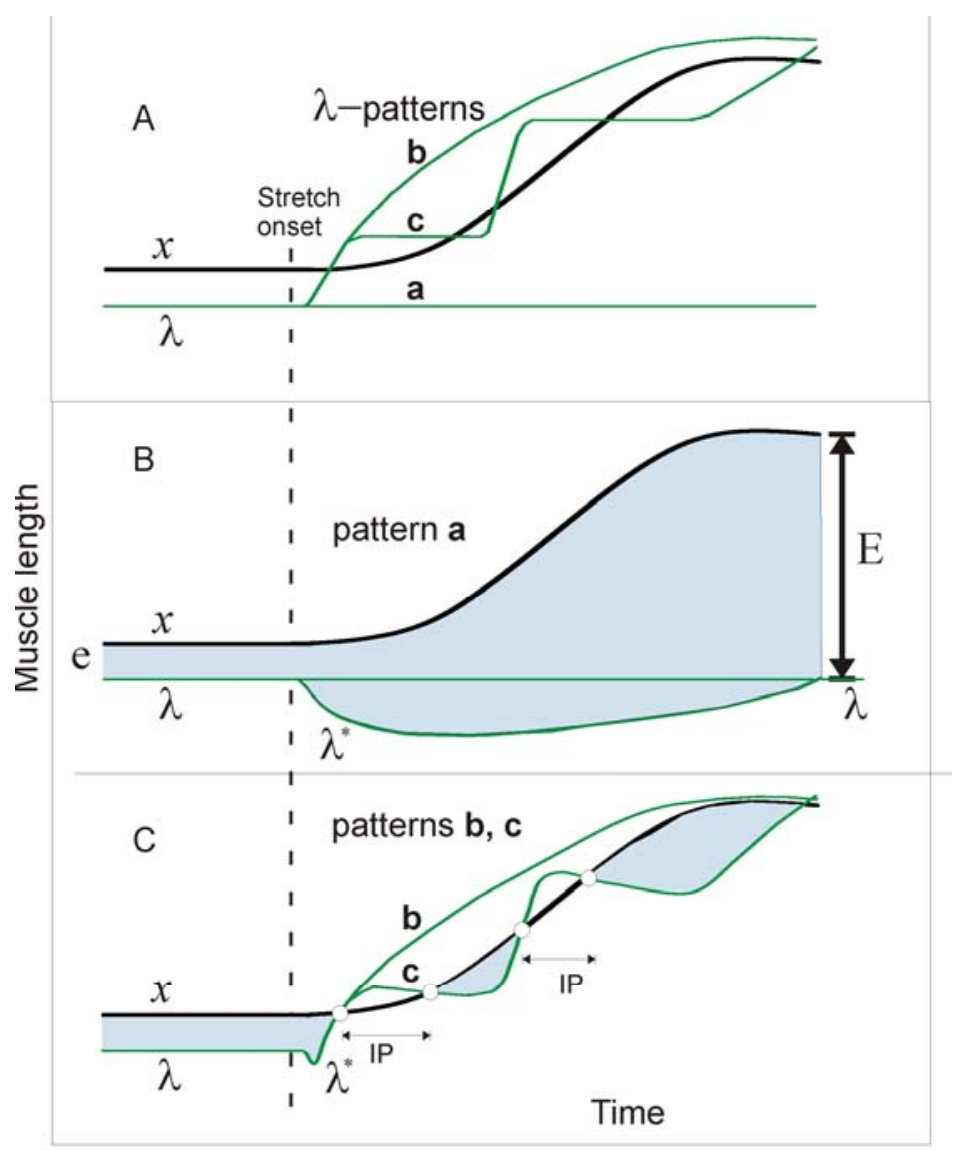

Figure 4

Qualitative explanations of results in terms of threshold position control. A: 3 possible patterns (ac) of central changes in the threshold muscle length $(\lambda)$ in response to passive changes in muscle length $(x)$ are shown. Pattern a: $\lambda$ is constant, i.e., the control system tolerates the muscle stretch without intervening. Pattern $b$ : the system continuously increases $\lambda$ to make it overrun and remain ahead of $x$ during the entire muscle stretch. Pattern $c$ : in response to the stretch onset, the system triggers a set of discrete ramp-and-hold increases in $\lambda$, each time overrunning the muscle stretch. $B$ : dynamics of muscle activation during pattern a. Muscle activation is roughly proportional to the difference between $x$ and $\lambda^{*}$ (vertical distance between upper and lower curves). Before the stretch onset, muscle activation is defined by distance $e$. Although $\lambda$ remains constant, the dynamic threshold $\left(\lambda^{*}\right)$ decreases with stretch velocity (i.e., deviates down from $\lambda$ line). After the stretch onset, the shaded area below the $\lambda$ line represents the phasic, velocity-dependent SR component; the shaded area above the $\lambda$ line is the tonic, length-dependent SR component. After the end of stretching, the phasic component vanishes, but the tonic component (E) of muscle activation remains and substantially exceeds the initial, pre-stretch activation level $(E>>e)$. C: during pattern $b$, the phasic component of the SR is suppressed and the muscle becomes deactivated, thus ceasing active muscle resistance during the entire muscle stretch. During pattern $c$, the phasic SR component and the ongoing muscle activation are also suppressed, producing an IP. During the holding phase of each $\lambda$ ramp, the continuing stretching reaches threshold $\lambda^{*}$ and the muscle is reactivated, but generates much less activity (defined by the small shaded areas below curve $x$ ) than during pattern $a$, thus minimizing active resistance to the muscle stretch. After learning to generate pattern $c$, the system can use it, without further learning, to minimize resistance to stretching of preliminary non-stretched muscles (generalization of learning). Reproduced with permission from Turpin et al. (2016). Copyright Springer. 
Note that, in shifting the spatial threshold, the system does not eliminate the SR as such - it just resets ("re-addresses") it to a new range of positions at which the SR remains fully functional. This central strategy has been shown to underlie the control of posture, movement, isometric torque and motor learning. It also solves several classical problems in motor control (Feldman et al., 2011; Feldman, 2015). For example, the corticospinal system was found to be directly involved in feed-forward setting and resetting of spatial thresholds in the control of the wrist position and movement (Ilmane et al., 2013; Raptis et al., 2010; Sangani et al., 2011). Studies in animals suggest that all descending systems act on spinal mechanisms by setting spatial muscle activation thresholds and, as a consequence, specifying the position at which muscle active torque/length characteristics become effective (Feldman and Orlovsky, 1972). Thus, regulation of SRs is explained in the context of threshold position control that also underlies control of posture and movement.

\section{Control of movement after central nervous system lesions}

Central nervous system lesions cause disruptions in the ability of central structures to specify where, in a spatial domain or FR, MNs can be activated. This disruption leads to the appearance of several types of sensorimotor impairment in patients with neurological lesions including, but not limited to a stroke, traumatic brain lesions and cerebral palsy. Experimental data suggest that muscle weakness, spasticity and changes in muscle activation patterns during voluntary movement (e.g., diminished MN recruitment, changes in EMG/force relationships, lack of reciprocal activation, excessive coactivation, etc.) are all consequences of the same pathophysiology, illustrated by the model shown in Figure $1 \mathrm{~B}-\mathrm{a}$ deficit in the specification and regulation of SR thresholds (SRTs) within the spatial domain.

Neurological lesions affect the function of damaged cortical neurons as well as the balance of excitatory and inhibitory intra- and interhemispheric connections and their descending tracts (Burke et al., 2014; Cheung et al., 2016). Together with altered central processing of afferent information, damage to descending pathways leads to deficits in the specification and regulation of SRTs of individual muscles. An important consideration is that the SRT is the initial angle at which MNs begin to be recruited according to their length/tension relationships so that below or at the SRT, the system is in the resting state (i.e., when there is no muscle activation and movement). The SRT, called the tonic stretch reflex threshold (TSRT), can be evaluated by taking into account the velocitydependent nature of the SRT. It can be estimated from the relationship between the velocity of the stretch and the angle at which EMG of the stretched muscle begins to appear (velocity/angle relationship).

\section{SRTs in single joint systems}

Spatial locations of TSRTs were located in elbow flexors and extensors in patients with arm hemiparesis after a stroke by producing a series of stretches of the muscle at different velocities while the subject was instructed to relax (at rest). In healthy individuals, even stretches at high velocities $(>300 \%$ s) may not evoke any reflex activity in passive elbow muscles. However, stretches at velocities as low as $8 \%$ in patients with a stroke could evoke a stretch response in the muscle. A robust finding is that the angular threshold at which the stretch response occurs decreases with the speed of the stretch (e.g., faster stretches evoke a stretch reflex at a shorter muscle length).

This velocity-dependent threshold is termed the "dynamic stretch reflex threshold' or DSRT to distinguish it from the TSRT. Indeed, at zero-velocity DSRT coincides with TSRT. By performing a series of stretches at different velocities, the velocity/angle relationships of the stretch reflex responses in a series of patients with hemiplegia was characterized (Figure 5; Jobin and Levin, 2000; Levin et al., 2000). It was found that at rest, the TSRT of spastic muscles lay abnormally within the joint range, suggesting that the damaged nervous system could not regulate the TSRT beyond the biomechanical range as in healthy subjects, in order for muscle relaxation to occur. Clinically, the deficit in TSRT regulation leads to the appearance of spasticity when the muscle is stretched passively by an examiner or actively by voluntary contraction of the antagonist. The spasticity has a spatial characteristic. It is present at all muscle lengths or 
joint ranges beyond the TSRT value, an angular zone called the 'spasticity zone' (Figure 1B).

TSRTs were measured in agonist and antagonist muscles of the elbow in patients with post-stroke hemiparesis. Each patient showed a unique pattern of locations of flexor and extensor TSRTs that was roughly related to the severity of their clinical impairment (Levin et al., 2000). It was found that when the TSRT values in antagonist muscles did not overlap, the angular zone between them was characterized by the ability of the patient to activate flexor and extensor muscles with normal EMG patterns. Thus, when elbow flexion was initiated in a joint range between the two thresholds, muscle activation was reciprocal, as typically seen in healthy individuals. However, if the TSRT of either the flexor or extensor was surpassed during the movement, the rest of the movement would be characterized by abnormal muscle coactivation.
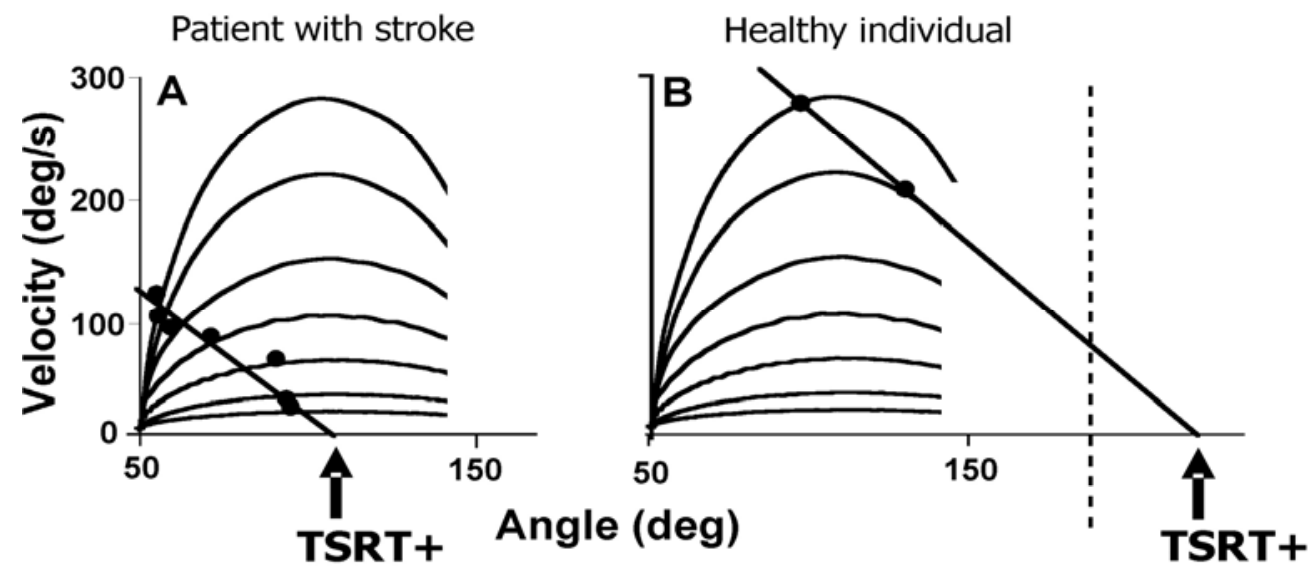

\section{Figure 5}

Experimentally determined relationship between velocity of the stretch and the stretch reflex threshold in elbow flexors in one patient with a stroke $(A)$ and a healthy subject (B). Thresholds were determined as the velocity and angle corresponding to the onset of EMG activity in the biceps brachii during the stretch of the muscle when the subject was at rest. Velocity-dependent velocity/angle thresholds (black dots) varied with the speed of the stretch with slower stretches (lower lines) resulting in EMG activation occurring at higher joint angles than faster stretches (top curved lines). In the healthy individual, no stretch responses were evoked at slower velocities. Locations of the TSRT+ within $(A)$ or beyond $(B)$ the angular range of the joint were identified by extrapolation of a linear regression line through all the velocity-dependent thresholds to zero velocity. 

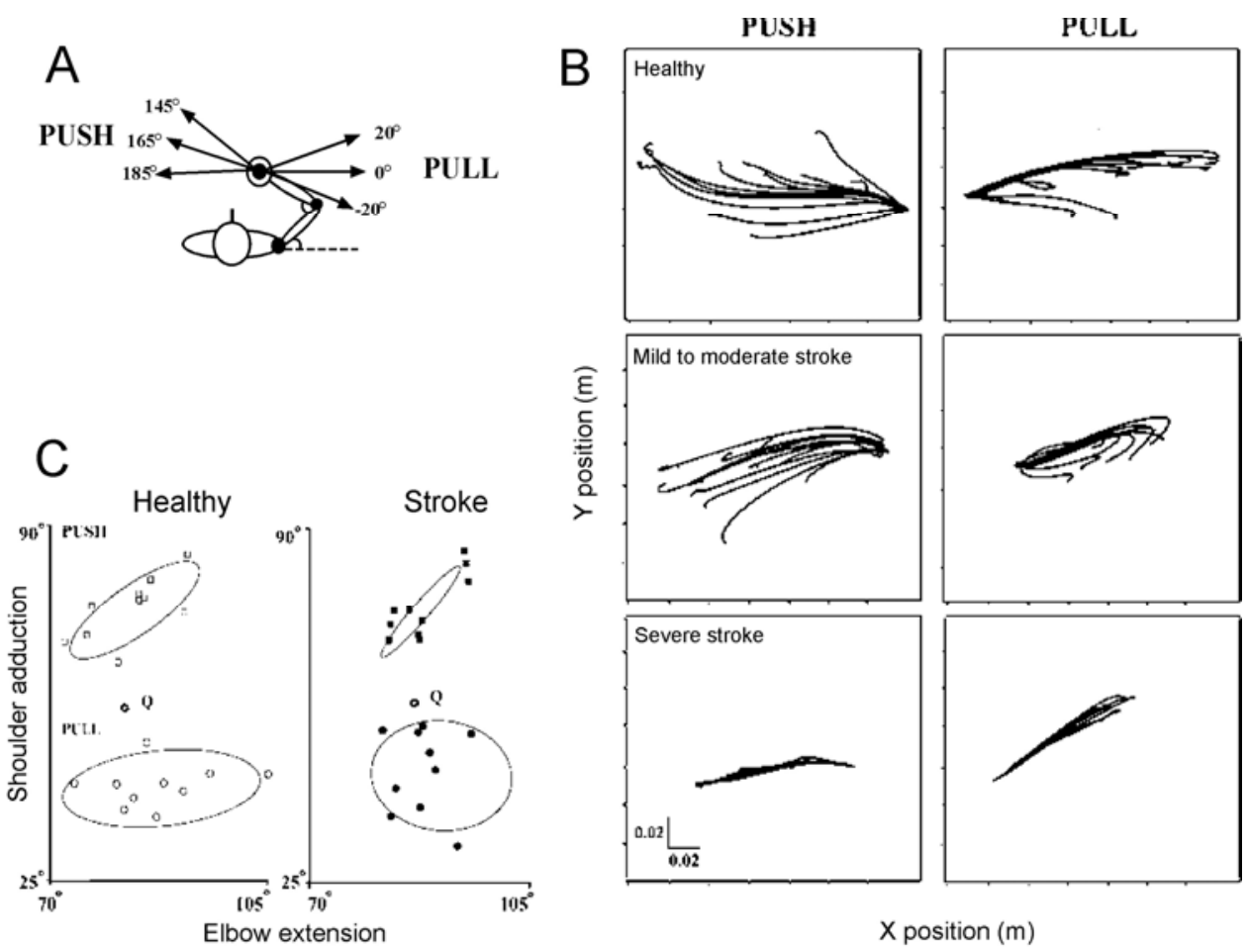

X position (m)

\section{Figure 6}

Experimental design to identify referent hand positions. A: The subject sat in front of a computer screen with the trunk supported and the right arm attached to the handle of a double-joint manipulandum controlled by two torque motors. They were instructed to match the force of the motors by pushing the handle to the left $\left(165^{\circ}\right)$ or pulling it to the right $\left(0^{\circ}\right)$. For both initial conditions, 3 directions of the final force: $0^{\circ}, 20^{\circ}$ and $-20^{\circ}$ with respect to the direction of the initial force were used. B. Examples of mean trajectories for each of the 12 unloading conditions for the push (left panels) and pull (right panels) directions in a healthy subject (top) and in participants with mild to moderate (middle) and severe (bottom) arm motor impairment. C. Referent arm configurations in elbow vs shoulder angle coordinates for the push (squares) and pull (circles) directions for healthy subjects (open symbols) and participants with a stroke (filled symbols). The 95\% confidence ellipse for each group of points is indicated. Data are shown in reference to the initial configuration of the arm (open hexagons, $Q$ ). Figure shows that the range of regulation of $R$ for each direction was restricted in patients with a stroke. Adapted with permission from Mihaltchev et al. (2005).

\section{SRTs in multi-joint systems}

Using a similar methodology for estimating TSRTs in single joints, the SRT concept was generalized to the multi-joint case for movements of the double-joint arm in patients with a stroke (Musampa et al., 2007). EMG activity from 4 elbow and 2 shoulder muscles was recorded. Muscles of interest spanned one (brachioradialis, anconeus, anterior deltoid, posterior deltoid) or two joints (biceps brachii, triceps brachii). Activity was evoked by applying 
a quasi-static $\left(<5^{\circ} / \mathrm{s}\right)$ stretch of elbow flexors/extensors and during slow voluntary elbow flexion/extension movement through full range. Stretches and active movements were initiated from full elbow flexion or extension. To determine the influence of the shoulder position on elbow muscle responses, the shoulder was placed in 3 different initial positions $\left(60^{\circ}, 90^{\circ}, 145^{\circ}\right.$ horizontal abduction) that resulted in changes in the initial lengths of muscles spanning both one or both joints. TSRT angles obtained by a passive muscle stretch were compared with those at which the respective muscles became active during slow voluntary elbow movements. The angular locations of TSRTs of elbow flexors and extensors varied with changes in the shoulder angle in all subjects with hemiparesis, but could not be identified in healthy subjects when muscles were relaxed. Similarly to the results of TSRT testing in the single, elbow joint, the limitations in TSRT regulation in the shoulder-elbow system resulted in a subdivision of possible shoulderelbow arm configurations into two spatial areas, one in which spasticity was present (spasticity zone) and another in which it was absent (active control zone). Spatial spasticity zones differed for various muscles in different patients but, taken together, the zones occupied a large part of the double-joint space in each patient. These results suggest that the shape of the boundary between the spasticity and active control zones depended on reflex inter-joint and inter-muscular interactions. In patients with neurological deficits, attempts to make functional arm movements are likely limited when the gesture requires that a particular joint or joint combination reaches and goes outside the borders of their active control zones.

As suggested above, multi-joint movements result from the specification of a referent configuration of the body, a centrally specified body posture at which multiple muscles of the body reach their SRTs, or in the presence of agonist-antagonist coactivation, a body posture at which active muscle torques at all body segments reach minimal values (Feldman, 2015, 2016). The activity of muscles and forces required for movements emerge according to the difference between the actual and referent body configurations. We tested this notion in patients with a stroke who had sustained a lesion in one hemisphere of the brain. We identified the referent arm configuration of the arm during a task in which the subject had to bring the arm to the target position (voluntary action) and to maintain the position during sudden unloading (involuntary responses), in healthy individuals and in patients with stroke related hemiparesis (Mihaltchev et al., 2005). With the right arm secured to a double-joint manipulandum, subjects held their arm in an initial configuration by matching a force applied to their hand via a handle that was equivalent to $30 \%$ of their maximal voluntary force in that position. The external force pulled the hand to the left $\left(165^{\circ}\right)$ or pushed it to the right $\left(0^{\circ}\right)$. Then, the load was unexpectedly partially or completely removed. For both the initial conditions, 3 directions of the final force $\left(0^{\circ},+20^{\circ}\right.$ and $\left.-20^{\circ}\right)$ with respect to the direction of the initial force were used. Subjects were instructed not to intervene when the load was changed, but to let their arm move naturally and come to rest in a new final position. Partial removal of the load resulted in distinct final hand positions associated with unique shoulder-elbow configurations and joint torques for both groups of subjects. The net static torque at each joint before and after unloading was represented as a function of the two joint angles describing a planar surface or invariant characteristic in $3 \mathrm{D}$ torque / angle coordinates. For each initial condition, the referent arm configuration was identified as the combination of elbow and shoulder angles at which the net torques at the two joints were zero. These configurations differed for different initial conditions. The identification of the referent configuration was possible for all healthy participants and for most individuals with hemiparesis suggesting that they preserved the ability to adapt their central commands - the referent arm configurations - to accommodate changes in external load conditions. Although the basic response patterns were preserved in the patient group, they had a more restricted range of hand trajectories following unloading (Figure 6), increased instability around the final endpoint position, altered patterns of elbow and shoulder muscle coactivation and differences in the dispersion of referent configurations in elbow-shoulder joint space compared to healthy individuals. These results suggest that problems in the specification of the 
referent configuration may be responsible for the inability of some individuals with a stroke to produce coordinated multi-joint movements. The present work adds three findings to the motor control literature concerning the consequences of the stroke - non-significant torque / angle regression in some subjects, narrower range of final arm configurations and instability around the final position.

\section{Association with clinical measurement of spasticity}

Abnormally regulated TSRTs have been described in the elbow and ankle in adults and children with spasticity (Blanchette et al., 2016; Jobin and Levin, 2000; Levin et al., 2000). Current measures of spasticity are not based on the physiological definition of the disorder and have shown low test-retest reliability. Indeed, the current 'gold standard' clinical spasticity measure (modified Ashworth scale, Bohannon and Smith, 1987) subjectively quantifies the amount of resistance of a muscle to a passive stretch and does not take into account the velocitydependence of the response. Thus, it lacks construct validity. The SRT measure, in contrast, is based on the physiological definition of spasticity and a model of disordered motor control (Lance, 1980; Levin and Feldman, 1994). The concept of threshold control was integrated in the report of a consensus panel of experts on hypertonic disorders in children and published in Pediatrics in 2003 (Sanger et al., 2003). The measure can be used to diagnose not only where in the angular range of the joint spasticity occurs, but also the presence of abnormal muscle activation patterns during voluntary movement (Levin et al., 2000). Studies have demonstrated that it has good inter-rater and test-retest reliability for elbow and ankle spasticity in patients with a stroke and cerebral palsy (Blanchette et al., 2016; Calota et al., 2008; Calota and Levin, 2009; Jobin and Levin, 2000) and that it distinguishes between spasticity due to a stroke and rigidity in patients with Parkinson Disease (Mullick et al., 2013).

Clinical relevance: The studies in singleand double-joint arm systems performed at rest and during voluntary efforts are based on the unified theory of disordered motor control within the referent configuration theory. Our findings to date suggest that the TSRT is a valid and reliable measure of disordered motor control and spasticity. Future research will focus on determining the responsiveness of the measure to change due to pharmacological and physical treatment interventions and its relationship with neurological recovery.

\section{Acknowledgements}

MFL holds a Tier 1 Canada Research Chair in Motor Recovery and Rehabilitation. Studies supported by NSERC, CIHR. Canadian Foundation for Innovation and Heart and Stroke Foundation of Canada.

\section{References}

Andronov AA, Chaikin SE. Theory of oscillations. Princeton, NJ: Princeton University Press (Original work in Russian published in 1937); 1949

Asatryan DG, Feldman AG. Functional tuning of the nervous system with control of movement or maintenance of a steady posture: I. Mechanographic analysis of the work of the joint or execution of a postural task. Biophysics, 1965; 10: 925-934

Blanchette AK, Mullick AA, Moin-Darbari K, Levin MF. Tonic stretch reflex threshold as a measure of ankle plantar-flexor spasticity after stroke. Phys Ther, 2016; 96: 687-695

Bohannon RW, Smith MB. Interrater reliability of a modified Ashworth scale of muscle spasticity. Phys Ther, 1987; 67(2): 206-207

Burke E, Dodakian L, See J, McKenzie A, Riley JD, Le V, Cramer SC. A multimodal approach to understanding motor impairment and disability after stroke. J Neurol, 2014; 261(6): 1178-1186.

Calota A, Feldman AG, Levin MF. Spasticity measurement based on tonic stretch reflex threshold in stroke 
using a portable device. Clin Neurophysiol, 2008; 119: 2329-2337

Calota A, Levin MF. Tonic stretch reflex threshold as a measure of spasticity: implications for clinical practice. Top Stroke Rehabil, 2009; 16: 177-188

Cheung DK, Climans SA, Black SE, Gao F, Szilagyi GM, Mochizuki G. Lesion characteristics of individuals with upper limb spasticity after stroke. Neurorehabil Neural Rep, 2016; 30: 63-70

Evarts EV, Tanji J. Gating of motor cortex reflexes by prior instruction. Brain Res, 1974; 71: 479-494

Fedirchuk B, Dai Y. Monoamines increase the excitability of spinal neurones in the neonatal rat by hyperpolarizing the threshold for action potential production. J Physiol, 2004; 557: 355-361

Feldman AG. Once more on the equilibrium-point hypothesis (lambda model) for motor control. $J$ Mot Behav, 1986; 18: 17-54

Feldman AG. Referent control of action and perception: Challenging conventional theories in behavioral neuroscience. New York: Springer; 2015

Feldman AG. Active sensing without efference copy: referent control of perception.

J Neurophysiol, 2016 [Epub ahead of print]

Feldman AG, Krasovsky T, Banina MC, Lamontagne A, Levin MF. Changes in the referent body location and configuration may underlie human gait, as confirmed by findings of multi-muscle activity minimizations and phase resetting. Exp Brain Res, 2011; 210: 91-115

Feldman AG, Orlovsky GN. The influence of different descending systems on the tonic stretch reflex in the cat. Exp Neurol, 1972; 37: 481-494

Glansdorff P, Prigogine I. Thermodynamics theory of structure, stability and fluctuations. London: WileyInterscience; 1971

Heckman CJ, Johnson M, Mottram C, Schuster J. Persistent inward currents in spinal motoneurons and their influence on human motoneuron firing patterns. Neuroscientist, 2008; 14: 264-275

Horak F, Diener H, Nashner L. Influence of central set on human postural responses. J Neurophysiol, 1989; 62: $841-853$

Hultborn H. Spinal reflexes, mechanisms and concepts: from Eccles to Lundberg and beyond. Prog Neurobiol, 2006; 78: 215-232

Ilmane N, Sangani S, Feldman AG. Corticospinal control strategies underlying voluntary and involuntary wrist movements. Behav Brain Res, 2013; 236: 350-358

Jobin A, Levin MF. Regulation of stretch reflex threshold in elbow flexors in children with cerebral palsy: a new measure of spasticity. Dev Med Child Neurol, 2000; 42(8): 531-540

Krawitz S, Fedirchuk B, Dai Y, Jordan LM, McCrea DA. State-dependent hyperpolarization of voltage threshold enhances motoneuron excitability during fictive locomotion in the cat. J Physiol, 2001; 532: 271-281

Lance JW. The control of muscle tone, reflexes, and movement: Robert Wartenberg Lecture. Neurology, 1980; 30: 1303-1313

Levin MF, Feldman AG. The role of stretch reflex threshold regulation in normal and impaired motor control. Brain Res 1994; 657(1-2): 23-30

Levin MF, Selles RW, Verheul MHG, Meijer OG. Deficits in the coordination of agonist and antagonist muscles in stroke patients: implications for normal motor control. Brain Res, 2000; 853: 352-369

Matthews PB. A study of certain factors influencing the stretch reflex of the decerebrate cat. J Physiol, 1959; 147: 547-564 
Mihaltchev P, Archambault PS, Feldman AG, Levin MF. Control of double-joint arm posture in patients with unilateral brain damage. Exp Brain Res, 2005; 163: 468-486

Mullick AA, Musampa NK, Feldman AG, Levin MF. Stretch reflex spatial threshold measure discriminates between spasticity and rigidity. Clin Neurophysiol, 2013; 124: 740-751

Musampa NK, Mathieu PA, Levin MF. Relationship between stretch reflex thresholds and voluntary arm muscle activation in patients with spasticity. Exp Brain Res, 2007; 181: 579-593

Nashner LM. Adapting reflexes controlling the human posture. Exp Brain Res, 1976; 26: 59-72

Raptis H, Burtet L, Forget R, Feldman AG. Control of wrist position and muscle relaxation by shifting spatial frames of reference for motoneuronal recruitment: possible involvement of corticospinal pathways. J Physiol, 2010; 588: 1551-1570

Rothwell JC, Day BL, Berardelli A, Marsden CD. Habituation and conditioning of the human long latency stretch reflex. Exp Brain Res, 1986; 63: 197-204

Sangani SG, Raptis HA, Feldman AG. Subthreshold corticospinal control of anticipatory actions in humans. Behav Brain Res, 2011; 224(1): 145-154

Sanger TD, Delgado MR, Gaebler-Spira D, Hallett M, Mink JW. Task Force on Childhood Motor Disorders. Classification and definition of disorders causing hypertonia in childhood. Pediatrics, 2003; 111: e89e97

Sillar KT. Spinal pattern generation and sensory gating mechanisms. Curr Opin Neurobiol, 1991; 1: 583-589

Stein RB, Capaday C. The modulation of human reflexes during functional motor tasks. Trends Neurosci, 1988; 11: 328-332

Stuart DG. Integration of posture and movement: contributions of Sherrington, Hess, and Bernstein. Hum Mov Sci, 2005; 24: 621-643

Turpin NA, Levin MF, Feldman AG. Implicit learning and generalization of stretch response modulation in humans. J Neurophysiol, 2016; 115(6): 3186-3194

von Holst H. Relations between the central nervous system and the peripheral organs. Br J Anim Behav, 1954; 2: 89-94

Wei K, Glaser JI, Deng L, Thompson CK, Stevenson IH, Wang Q, Hornby TG, Heckman CJ, Kording KP. Serotonin affects movement gain control in the spinal cord. J Neurosci, 2014; 34: 12690-12700

\section{Corresponding author:}

\section{Dr. Mindy F. Levin, PhD}

School of Physical and Occupational Therapy

McGill University

3654 Promenade Sir William Osler

Montreal, Quebec.Canada

H3S 2J4

Phone: +514-398-3994

E-mail: mindy.levin@mcgill.ca; feldman@med.umontreal.ca 